\title{
Effectiveness of temporary positive expiratory pressure (T-PEP) at home and at hospital in patients with severe chronic obstructive pulmonary disease
}

\author{
Valentina Mascardi ${ }^{1}$, Bruna Grecchi ${ }^{2}$, Cornelius Barlascini ${ }^{3}$, Paolo Banfi ${ }^{4}$, Antonello Nicolini ${ }^{1}$ \\ ${ }^{1}$ Respiratory Diseases Unit, Hospital of Sestri Levante, Sestri Levante, Italy; ${ }^{2}$ Physical Medicine and Rehabilitation, Chiavari, Italy; ${ }^{3}$ Hygiene and \\ Health Medicine Hospital of Sestri Levante, Sestri Levante, Italy; ${ }^{4}$ Pulmonary Diseases Unit, Don Gnocchi Foundation IRCCS, Milan, Italy \\ Contributions: (I) Conception and design: A Nicolini, V Mascardi; (II) Administrative support: B Grecchi, C Barlascini; (III) Provision of study \\ materials or patients: A Nicolini, V Mascardi; (IV) Collection and assembly of data: A Nicolini, V Mascardi, P Banfi, B Grecchi; (V) Data analysis \\ and interpretation: P Banfi, C Barlascini; (VI) Manuscript writing: All authors; (VII) Final approval of manuscript: All authors. \\ Correspondence to: Antonello Nicolini, MD. Respiratory Diseases Unit, General Hospital, via Terzi 43 - 16039 Sestri Levante, Italy. \\ Email: antonellonicolini@gmail.com.
}

Background: Temporary positive airway pressure (T-PEP) is a tool recently introduced in the treatment of chronic obstructive pulmonary disease (COPD) or bronchiectasis. It demonstrated encouraging results also in severe COPD patients. The aim of this study is verify if adding T-PEP to best bronchodilator therapy both in clinic and home administering could reduce disease exacerbations and improve lung function in patients with severe COPD.

Methods: A total of 142 patients with severe COPD (FEV1 <50\%) were enrolled; 120 were randomized in three groups: a group treated with T-PEP at home, a group with T-PEP at hospital and a group with medical therapy only (control group). Number of acute exacerbations COPD (AECOPD) after 1 month and 3 months were the primary outcomes. Secondary outcomes were changes in respiratory function parameters (FVC, FEV1, TLC, RV), arterial blood gases, dyspnea and health status assessment scales (Modified Medical Research Council (MMRC), Breathlessness, Cough and Sputum scale (BCSS) and COPD Assessment Test (CAT). The time of daily use of the T-PEP was registered as well as its acceptance using a Likert scale.

Results: Ninety-nine patients completed the study. Both the groups who used T-PEP showed a statistical lower number of AECOPD after 1 month and 3 months $(\mathrm{P}<0.01)$. Some respiratory functional parameters improved in the two groups treated with T-PEP (FVC, FEV1, RV) $(\mathrm{P}<0.02)$ and dyspnea and health status assessment scales (MMRC, BCSS, CAT) $(\mathrm{P}<0.04 ; \mathrm{P}<0.01 ; \mathrm{P}<0.009)$. The time of daily using was similar in the two T-PEP groups. Patients treated at home showed a greater acceptance than those treated at hospital (Likert scale 4.7 vs. 5.9) $(\mathrm{P}<0.01)$.

Conclusions: Patients treated with T-PEP showed a lower number of AECOPD. T-PEP improves functional respiratory parameters and improves dyspnea and health status assessment scales. No adherence difference in hospital and home treatment was found. Patients preferred home treatment.

Keywords: Severe chronic obstructive pulmonary disease; temporary expiratory pressure (T-PEP); home administering; hospital administering

Submitted Jul 08, 2016. Accepted for publication Sep 12, 2016.

doi: $10.21037 /$ jtd.2016.10.69

View this article at: http://dx.doi.org/10.21037/jtd.2016.10.69 


\section{Introduction}

Mucus hypersecretion and impaired mucociliary clearance is prevalent in many patients with chronic obstructive pulmonary disease (COPD), and contributes significantly to the morbidity and mortality of this disease (1-4). Despite of the need for efficacious, convenient and safe treatment for mucus hypersecretion, current choices are few with limited data to support their efficacy in $\operatorname{COPD}(1,5,6)$. The use of positive expiratory pressure (PEP) devices for COPD patients is increasing in the last years because of the beneficial effects on lung function (similar to conventional chest physiotherapy), the high level of acceptance and adherence by patients and possibility of home use (avoiding hospital treatment) $(4,7,8)$. The greatest number of studies using PEP was performed in cystic fibrosis patients $(4,7)$. They have shown that this technique often improves lung function parameters, increases secretion detachment with quantity of sputum expectorated and improves dyspnea (7-9). Temporary expiratory pressure (T-PEP) is a device which applies an expiratory pressure $\leq 1 \mathrm{cmH}_{2} \mathrm{O}$ only for a fraction of the expiratory phase (7-9). This increase in low pressure is created through a pulsatile flow approximately $42 \mathrm{~Hz}$ in frequency (8). T-PEP works by detaching and removing secretions from the peripheral airways, improving pulmonary function testing (including reduction in air trapping) $(7,10)$. Some studies on COPD patients have shown encouraging results and improvement in pulmonary function parameters, bronchial encumbrance, dyspnea and quality of life $(4,8,10)$. No study is available on home use of T-PEP for patients with chronic hyper-secretive disorders.

This study aimed to evaluate feasibility, effectiveness and acceptance of a two-week treatment with T-PEP at hospital and at home in patients with severe COPD [forced expiratory volume 1 second (FEV1)<50\%].

\section{Methods}

This is a fifteen weeks parallel randomized controlled study that aimed to verify the effectiveness and the acceptance of a T-PEP treatment administered at hospital or at home in patients with severe COPD (FEV1 <50\%) (GOLD stages III and IV).

\section{Patients}

One hundred and forty-two patients with severe COPD [Forced expiratory volume 1 second (FEV1) $<50 \%$ ] was recruited between September 2012 and November 2014. Inclusion criteria were: at least 35 years of age, presence of chronic bronchitis, presence of airway obstruction at spirometry (GOLD stage III-IV). Exclusion criteria were: exacerbation of COPD or hospitalization for COPD within 8 weeks prior to recruitment, predominant asthma or bronchiectasis by clinical assessment, presence of tracheostomy, inability to comply with study procedures or absence of written informed consent.

\section{Study design}

The study consisted of a screening visit, two weeks of intervention-free run it and optimization of medical therapy, a randomization visit and then on treatment clinic visit at 1, 2, 4, 8, 15 weeks. Participants who met inclusion criteria on screening visit were enrolled and instructed to registered all data during the study period. A randomization visit was done within 2 weeks of the enrollment visit. A randomization schedule was generated by a statistician not involved in the study using an online random permutation generator from htpp://www.randomization.com. The randomization assignment was provided to the recruiting physicians in sealed envelopes. The patients and the investigators who carried out the study data analysis were blinded to the patients' treatment assignments. All the patients were being treated with association of inhaled $\beta-2-$ agonist plus anti-muscarinic drug. Eighteen patients had chronic respiratory insufficiency had been treated with long-term oxygen. One hundred and forty-two patients were enrolled and 120 included in the study (twentytwo were excluded: 8 because of concomitant bronchial asthma, 6 bronchiectasis, 4 inability to perform 6 -minute walk test (6MWT), 4 absence of written informed consent). The randomized patients were divided into three groups (40 patients for each group): one group was treated with T-PEP at hospital (Hosp- TPEP), one group was treated with T-PEP at home (H-TPEP) and one group with pharmacological therapy alone (control group) (Figure 1: patients' flow chart). All the eligible patients after the randomization were instructed by a physiotherapist or a specialized nurse on the use of T-PEP and acclimatization in a one-hour training period in the lung laboratory before definitive inclusion in the study protocol. The treatment lasted $30 \mathrm{~min}$ per session and was given twice daily (morning and late afternoon). The duration of the treatment for each group was fifteen days. The usage of T-PEP at home was assessed using a counter inside the instrument and a 


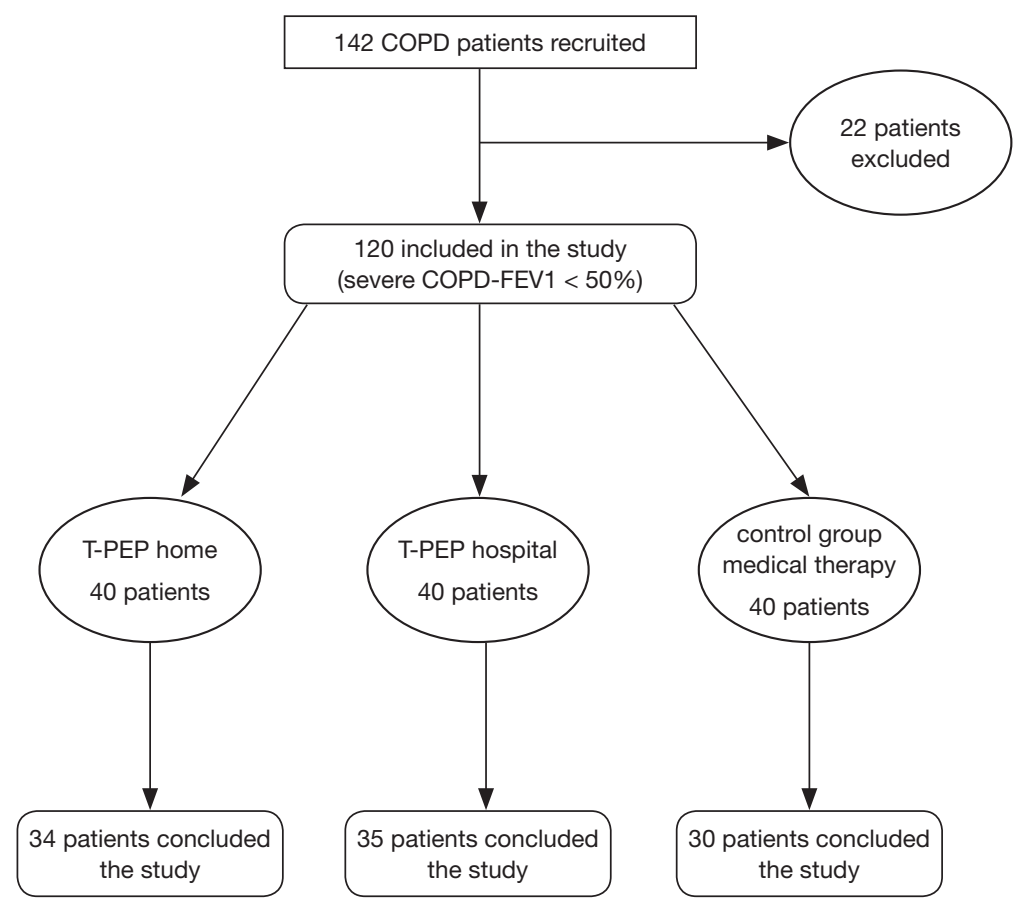

Figure 1 Patients' flow. T-PEP, temporary positive expiratory pressure.

questionnaire where each patient reported the duration of every session of therapy. The study was registered at Chinese Clinical Trials as Chi-CTR-TRC 15006662 , carried out according to Helsinki Declaration and every patient signed informed consent. The study was approved by Local Ethics Committee ASL4 Chiavarese, Chiavari, Italy Del.502/2013.

\section{Measurements and outcomes}

At enrolment, patients' anthropometric and physiological characteristics were recorded. Respiratory measurements included dyspnea, cough and sputum scales, as well as daily life activity evaluations, respiratory function testing and hematological parameters. Respiratory function tests were: [forced vital capacity (FVC), FEV1, FEV1/FVC\%, total lung capacity (TLC), residual volume (RV), diffusing lung capacity monoxide (DLCO), maximal inspiratory pressure (MIP), maximal expiratory pressure (MEP)], arterial blood gas analysis $(\mathrm{ABG})\left(\mathrm{PaO}_{2}, \mathrm{PaCO}_{2}, \mathrm{pH}\right)$ and hematological examinations: white and red cell counts, $\mathrm{C}$ reactive protein, $\beta$-globulins. Moreover, exercise capacity was evaluated with 6MWT. Dyspnea, cough and sputum and health status assessment were measured with the Breathlessness, Cough and Sputum Scale (BCSS) (11,12), COPD Assessment Test
(CAT) (13-15) and the Modified Medical Research Council (MMRC) Dyspnea Scale (15). Diagnosis and severity of COPD were confirmed using the GOLD Guidelines (16). Pulmonary function testing was performed with a computerized body plethysmography (VMax 20 PFT Sensor Medics, Yorba Linda, CA, USA), according to the ATS/ERS Guidelines (17). Inspiratory muscle strength was assessed by measuring the maximal inspiratory mouth pressure (MIP) at RV. Expiratory muscle strength was also measured as maximal expiratory mouth pressure (MEP) at TLC. The value obtained from the best of at least three efforts were used. All the measurements were obtained in upright position. Dynamic and static volumes were expressed as a percentage of their predicted value. Functional exercise capacity was recorded from the $6 \mathrm{MWT}$ according to the American Thoracic Society Guidelines (18). BCSS, CAT and MMRC were recorded each day to evaluate the daily variations in dyspnea, health status assessment and perceived sensation of bronchial encumbrance. Finally, we evaluated the acceptance of both tools using a Likert scale.

\section{Endpoints}

The primary outcome was the reduction of acute exacerbations (AECOPD) after one, and three months. 
AECOPD was defined as a worsening of symptoms (following Anthonisen classification) or healthcare utilization with commencement of antibiotics and/or corticosteroids $(19,20)$. Secondary outcomes improvement of lung function testing, ABG, exercise capacity (measured by $6 \mathrm{MWT}$ ) as well as dyspnea and health status assessment (MMRC scale, BCSS scale and CAT), and hematological examinations (white and red cell counts, $\mathrm{C}$ reactive protein, $\beta$-globulins). The use of the instrument (minutes per day) as well as the patient acceptance and preference using a seven point Likert questionnaire (self administered) was evaluated too.

\section{Statistics}

Clinical data were expressed as mean \pm standard deviation (SD) or median and $95 \%$ confidence interval (CI). The median change and IC difference between each treatment at baseline and at the end of the treatment was estimated using ANCOVA test. For continuous variables F-test was used and $\chi$-square test for categorical variables. For continuous variables reporting median value a non-parametric test (Kruskal-Wallis) was used. $\mathrm{P} \leq 0.05$ was considered statistically significant. Data analysis was performed using SPSS statistics software, IBM Analytics, NY, USA.

\section{Results}

Ninety-nine patients concluded the study, 21 dropped out (six in the Hospital TPEP group, five in the Home TPEP group and ten in the control group). The average age of enrolled patients was $71.5 \pm 4.4$ years in the HospTPEP group, $70.7 \pm 6.3$ in the H-TPEP group and $71.0 \pm 6.5$ in the control group. All patients showed similar clinical characteristics except for MIP. The baseline characteristics of the randomized patients are summarized in the Table 1.

\section{Primary outcomes}

Both Hosp-TPEP and H-TPEP group have statistically lower number of AECOPD after 1 month $(\mathrm{P}<0.03)$ and 3 months $(\mathrm{P}<0.003)$ compared to the control group. No intergroup difference between Hosp-TPEP and H-TPEP in reducing number of AECOPD (Table 2) was noted.

\section{Secondary outcomes}

Laboratory and Respiratory Function parameters Both the groups who used Hosp-TPEP and H-TPEP therapy had improvement in some respiratory functional parameters (FVC, FEV1 and TLC and DLCO with $\mathrm{P}<0.001$ both in Hosp TPEP and H TPEP) compared to the control group. RV improved only in the Hosp TPEP $(\mathrm{P}<0.02)$. Moreover, MIP, MEP and 6MWT improved significantly in the two TPEP groups $(\mathrm{P}<0.001)$, as well as $\mathrm{paO}_{2}(\mathrm{P}<0.02)$. No significant differences was found between two TPEP group treatments. No significant differences in the three groups concerning white and red cells count or $\mathrm{C}$ reactive protein or $\Upsilon$-globulins. The most significant results are shown in Table 3.

\section{Dyspnea and health status assessment scales}

Dyspnea and health status assessment scales also improved in the T-PEP groups compared to control groups: MMRC $\mathrm{P}<0.001$, BCSS $\mathrm{P}<0.001$ and CAT $\mathrm{P}<0.001$. There was a significant difference in CAT between Hosp-TPEP and H-TPEP $(\mathrm{P}<0.03)$ (see Table 3).

\section{Average duration of the treatment}

The average duration of treatment was similar in the two groups $[61.1 \pm 2.8 \mathrm{~min}($ Hosp-TPEP) vs. $61.2 \pm 2.9 \mathrm{~min}$ (H-TPEP)] (Table 4).

\section{Patients acceptance}

The patients preferred home treatment (Likert scale $5.2 \pm 0.97$ vs. $5.7 \pm 0.89)(\mathrm{P}>0.02)($ Table 4).

\section{Discussion}

There is a paucity of the modalities that have demonstrated efficacy in the treatment of hypersecretion and bronchial obstruction in COPD patients. Mechanical means to improve mucus clearance in hypersecretory lung conditions (including oscillatory pep devices, chest vibration and percussion and breathing techniques) have not been tested systematically in stable COPD (1). PEP works shifting the equal pressure point peripherally to maxime airflow and protect dynamic airway collapse, increasing expiratory time, alveolar ventilation and facilitating gas exchange $(7,20,21)$.

Several previous studies have shown that PEP and T-PEP techniques improve lung volumes, dyspnea and quality of life scales and speed up the improvement of bronchial encumbrance $(4,8,10)$. Only few studies have been performed on incidence of re-exacerbations in COPD patients, but none demonstrated the effectiveness of these techniques $(20,21)$. Our study is one of the first which evaluated exacerbations among outcomes and besides 
Table 1 Demographic, clinical and functional parameters at baseline in the three groups

\begin{tabular}{|c|c|c|c|c|c|}
\hline Characteristics & Total sample $(n=120)$ & Hosp TPEP $(n=40)$ & H TPEP $(n=40)$ & Control group $(n=40)$ & $P$ value \\
\hline Age (years) & $71.1(5.7)$ & $71.7(4.6)$ & $70.7(6.1)$ & $70.7(6.3)$ & 0.7 \\
\hline Male (\%) & $86(72.9)$ & $28(71.8)$ & $30(75)$ & $28(71.8)$ & 0.9 \\
\hline Weight (kg) & $74.7(11.7)$ & $74.4(11.6)$ & $74.4(11.2)$ & $75.5(12.6)$ & 0.9 \\
\hline BMI & $26.2(4.0)$ & $25.9(2.9)$ & $25.7(4.0)$ & $26.9(4.8)$ & 0.6 \\
\hline \multicolumn{6}{|c|}{ Clinical and functional parameters } \\
\hline $\mathrm{PaO}_{2}$ & $71.8(6.9)$ & $70.3(7.4)$ & $72.5(5.7)$ & $72.6(7.4)$ & 0.3 \\
\hline $\mathrm{PaCO}_{2}$ & $41.4(5.2)$ & $41.7(5.5)$ & 40.9 (3.6) & $41.5(6.2)$ & 0.8 \\
\hline FEV1 (\%) & $35.6(10.6)$ & $37.9(10.7)$ & $35.6(10.0)$ & $33.2(11.0)$ & 0.1 \\
\hline FEV1/FVC (\%) & $48(11.8)$ & $49.1(12.2)$ & $48(10.7)$ & $46.9(12.6)$ & 0.7 \\
\hline TLC (\%) & $124.5(30.3)$ & $128.6(35.2)$ & $123.3(27)$ & $121.5(28.4)$ & 0.6 \\
\hline RV (\%) & $183(150.23)$ & $172(150.22)$ & $194.5(147.26)$ & $195(150.263)$ & 0.6 \\
\hline DLCO (\%) & $60.3(15.4)$ & $63.6(15.2)$ & $59.5(13.7)$ & 57.7 (16.9) & 0.2 \\
\hline MIP & $10[7,15]$ & $12[9,18]$ & $9.5[7,12]$ & $9[6,12]$ & 0.04 \\
\hline MEP & $11[8,16]$ & $14[8,18]$ & $11.5[7.5,14.5]$ & $9[7,16]$ & 0.1 \\
\hline \multicolumn{6}{|l|}{ Scales } \\
\hline MMRC scale & $3[3,4]$ & $3[3,4]$ & $3[2,3]$ & $3[3,4]$ & 0.3 \\
\hline CAT score & $23[19,28]$ & $22[19,28]$ & $22.5[19,26.5]$ & $25[19,29]$ & 0.4 \\
\hline BCSS scale & $7[5,8]$ & $6[5,8]$ & $7[6,8]$ & $7[5,8]$ & 0.2 \\
\hline
\end{tabular}

Data are expressed as mean \pm standard deviation or median $\mathrm{Cl} 25-75$. P value $<0.05$; F-test for continuous variables and $\chi$ square for variables. For continuous variables reporting median value non-parametric test Kruskal-Wallis.

Table 2 Number of acute exacerbations of COPD (AECOPD) in the three groups

\begin{tabular}{|c|c|c|c|c|c|}
\hline Variables & Overall sample (\%) & \multicolumn{3}{|c|}{ Summary of primary outcomes (\%) } & $P$ value ${ }^{\circ}$ \\
\hline After 1 month & $13(13.1)$ & $3(8.8)$ & $2(5.7)$ & $8(26.7)$ & 0.03 \\
\hline After 3 months & $24(24.2)$ & $5(14.7)$ & $5(14.3)$ & $14(46.7)$ & 0.003 \\
\hline
\end{tabular}

${ }^{\circ}, \chi^{2}$ test. COPD, chronic obstructive pulmonary disease. 
Table 3 Median changes (95\% CI) of principal clinical, functional and quality of life parameters at baseline and at the end of the treatment in the three groups

\begin{tabular}{|c|c|c|c|c|c|c|c|}
\hline Variables & Hospital T-PEP [1] & Home T-PEP [2] & Control group [3] & $\begin{array}{c}\text { Overall test } \\
\mathrm{P} \text { value }\end{array}$ & \multicolumn{3}{|c|}{ Pairwise comparison $\mathrm{P}$ values ${ }^{\circ \circ}$} \\
\hline $\mathrm{PaO}_{2}$ & $3.1(2.3 ; 3.9)$ & $2.7(1.9 ; 3.5)$ & $1.5(0.7 ; 2.4)$ & 0.02 & 0.5 & 0.008 & 0.04 \\
\hline $\mathrm{PaCO}_{2}$ & $-1.1(-1.8 ;-0.4)$ & $-1.5(-2.2 ;-0.8)$ & $-0.3(-1 ; 0.5)$ & 0.07 & 0.5 & 0.1 & 0.02 \\
\hline $\mathrm{HCO}_{3}$ & $-0.7(-1.1 ;-0.4)$ & $-0.9(-1.2 ;-0.5)$ & $-0.3(-0.7 ; 0.1)$ & 0.07 & 0.6 & 0.1 & 0.02 \\
\hline FEV1 (\%) & $7.5(6 ; 9)$ & $7.2(5.7 ; 8.6)$ & $-2.9(-4.5 ;-1.3)$ & $<0.0001$ & 0.8 & $<0.0001$ & $<0.0001$ \\
\hline TLC (\%) & $-14.1(-18 ;-10.1)$ & $-13(-16.9 ;-9.1)$ & $5.1(0.9 ; 9.3)$ & $<0.0001$ & 0.7 & $<0.0001$ & $<0.0001$ \\
\hline $\mathrm{RV}(\%)$ & $-30(-51.7 ;-8.3)$ & $-19.2(-40.6 ; 2.2)$ & $7.6(-15.5 ; 30.7)$ & 0.06 & 0.5 & 0.02 & 0.09 \\
\hline DLCO (\%) & $4.5(3.6 ; 5.5)$ & $4.1(3.2 ; 5.1)$ & $-2.6(-3.6 ;-1.6)$ & $<0.0001$ & 0.6 & $<0.0001$ & $<0.0001$ \\
\hline 6MWT (mt) & $24.1(16.6 ; 31.5)$ & $33.3(25.9 ; 40.7)$ & $-4.2(-12.3 ; 3.9)$ & $<0.0001$ & 0.1 & $<0.0001$ & $<0.0001$ \\
\hline MMRC scale & $-0.7(-0.9 ;-0.6)$ & $-0.5(-0.7 ;-0.3)$ & $0.2(0 ; 0.4)$ & $<0.0001$ & 0.06 & $<0.0001$ & $<0.0001$ \\
\hline BCSS scale & $-3.2(-3.7 ;-2.8)$ & $-3.3(-3.7 ;-2.8)$ & $-0.8(-1.2 ;-0.3)$ & $<0.0001$ & 0.9 & $<0.0001$ & $<0.0001$ \\
\hline CAT score & $-7.9(-8.9 ;-6.9)$ & $-6.4(-7.4 ;-5.5)$ & $-1.5(-2.5 ;-0.4)$ & $<0.0001$ & 0.03 & $<0.0001$ & $<0.0001$ \\
\hline
\end{tabular}

Average change and confidence interval evaluated using ANCOVA F-test. median value (25-75 pct): $\mathrm{P}$ value: Kruskal-Wallis test. ${ }^{\circ}$, ANCOVA F-test; ${ }^{\circ}, t$-test. $6 \mathrm{MWT}, 6$-minute walk test.

Table 4 Average time of use and level of acceptance of hospital T-PEP group and home T- PEP group

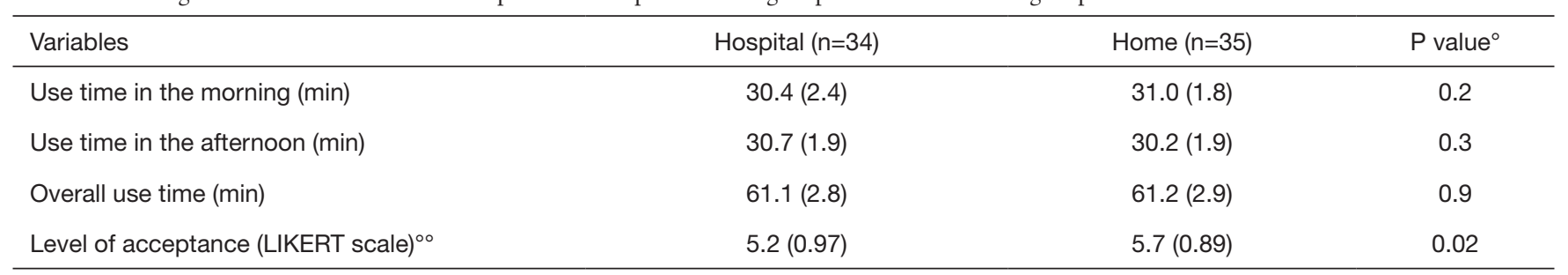

Data are expressed as mean (standard deviation). ${ }^{\circ}, t$-test; ${ }^{\circ}$, Likert scale $1-7$ points.

compared the results obtained in a usual and typical hospital environment to those obtained at home, where the treatment is self-administered. Some studies considered exacerbations between outcomes (20-23): only the study performed by Osadnik et al. is comparable to ours. This was performed on ninety-two AECOPD patients and evaluated the effect of PEP $\left(10-20 \mathrm{cmH}_{2} \mathrm{O}\right)$ on symptoms (MMRC dyspnea scale), exercise tolerance (6MWT), quality of life (St. George's Respiratory Questionnaire) and BCSS as well as number of AECOPD, hospital admission after eight weeks and six months follow-up. They found no benefit of PEP therapy on incidence of exacerbations, on symptoms or quality of life (20). Our study shows, on the contrary a lower number of exacerbation after one and three months compared to control group and an improvement of dyspnea scale, health status assessment scores, exercise tolerance and lung volumes. T-PEP, as previously demonstrated, decreases not only dynamic lung volumes but also static lung volumes and DLCO: it can mean a reduction of lung hyperinflation. This mechanism can explain an improvement of alveolar 
ventilation and an increase of $\mathrm{PaO}_{2}$.

This result has been reached only using this tool; it has never reported in any other study $(8,10)$. Finally, we tested the difference of outcome for self-administration at home or at in a hospital environment under medical or nurse supervision. The duration of the therapy at hospital and at home did not differ, but patients had a higher acceptance (Likert scale) at home. They preferred home treatment. Home treatment was feasible because the use of TPEP (UNIKO) device is very simple: one has to push once only a bottom (the patient cannot make a mistake and the instrument has a fixed positive pressure of $1 \mathrm{cmH}_{2} \mathrm{O}$ ).

\section{Limitations}

This study has three major limitations. First the observation period of the patients was short: our aim was to evaluate the short-term effect of an airway clearance device. Second the lack of information concerning exacerbation rate in previous years do not allow to state that the device is able to reduce AECOPD (but only that the patients treated with T-PEP had a reduced number of AECOPD compared to control group. Third, the high dropout rate could lead to an underestimation for comparison of adherence.

\section{Conclusions}

Patients treated with T-PEP have a lower number of AECOPD compared to patients treated with standard medical therapy. T-PEP improves functional respiratory parameters and improves dyspnea, health status assessment. No adherence difference in hospital and home treatment was found. Patients preferred home treatment.

\section{Acknowledgements}

The authors want to thank Dr. Giovanni Veronesi, Uninsubria University, Varese, Italy, for assistance in statistical analysis.

\section{Footnote}

Conflicts of Interest: The authors have no conflict of interest to declare.

Ethical Statement: The study was registered at Chinese Clinical Trials as Chi-CTR-TRC 15006662, carried out according to Helsinki Declaration and every patient signed informed consent. The study was approved by Local Ethics Committee ASL4 Chiavarese, Chiavari, Italy Del.502/2013.

\section{References}

1. Sethi S, Yin J, Anderson PK, et al. Lung flute improves symptoms and health status in COPD with chronic bronchitis: A 26 week randomized controlled trial. Clin Transl Med 2014;3:29.

2. Vestbo J, Prescott E, Lange P. Association of chronic mucus hypersecretion with FEV1 decline and chronic obstructive pulmonary disease morbidity. Copenhagen City Heart Study Group. Am J Respir Crit Care Med 1996;153:1530-5.

3. Miravitlles M, Calle M, Alvarez-Gutierrez F, et al. Exacerbations, hospital admissions and impaired health status in chronic obstructive pulmonary disease. Qual Life Res 2006;15:471-80.

4. Herrero-Cortina B, Vilaró J, Martí D, et al. Short-term effects of three slow expiratory airway clearance techniques in patients with bronchiectasis: a randomised crossover trial. Physiotherapy 2015. [Epub ahead of print].

5. Myers TR. Positive expiratory pressure and oscillatory positive expiratory pressure therapies. Respir Care 2007;52:1308-26; discussion 1327.

6. Rogers DF. Mucoactive agents for airway mucus hypersecretory diseases. Respir Care 2007;52:1176-93; discussion 1193-7.

7. Clini E. Positive expiratory pressure techniques in respiratory patients: old evidence and new insights. Breathe 2009;6:153-9.

8. Venturelli E, Crisafulli E, DeBiase A, et al. Efficacy of temporary positive expiratory pressure (TPEP) in patients with lung diseases and chronic mucus hypersecretion. The UNIKO® project: a multicentre randomized controlled trial. Clin Rehabil 2013;27:336-46.

9. Darbee JC, Ohtake PJ, Grant BJ, et al. Physiologic evidence for the efficacy of positive expiratory pressure as an airway clearance technique in patients with cystic fibrosis. Phys Ther 2004;84:524-37.

10. Nicolini A, Mollar E, Grecchi B, et al. Comparison of intermittent positive pressure breathing and temporary positive expiratory pressure in patients with severe chronic obstructive pulmonary disease. Arch Bronconeumol 2014;50:18-24.

11. Leidy NK, Rennard SI, Schmier J, et al. The breathlessness, cough, and sputum scale: the development of empirically based guidelines for interpretation. Chest 
2003;124:2182-91.

12. Leidy NK, Schmier JK, Jones MK, et al. Evaluating symptoms in chronic obstructive pulmonary disease: validation of the Breathlessness, Cough and Sputum Scale. Respir Med 2003;97 Suppl A:S59-70.

13. Dodd JW, Hogg L, Nolan J, et al. The COPD assessment test (CAT): response to pulmonary rehabilitation. A multicentre, prospective study. Thorax 2011;66:425-9.

14. Lee BY, Lee S, Lee JS, et al. Validity and Reliability of CAT and Dyspnea-12 in Bronchiectasis and Tuberculous Destroyed Lung. Tuberc Respir Dis (Seoul) 2012;72:467-74.

15. Cazzola M, Hanania NA, MacNee W, et al. A review of the most common patient-reported outcomes in COPD--revisiting current knowledge and estimating future challenges. Int J Chron Obstruct Pulmon Dis 2015;10:725-38.

16. Vestbo J, Hurd SS, Agustí AG, et al. Global strategy for the diagnosis, management, and prevention of chronic obstructive pulmonary disease: GOLD executive summary. Am J Respir Crit Care Med 2013;187:347-65.

17. Miller MR, Hankinson J, Brusasco V, et al. Standardisation of spirometry. Eur Respir J 2005;26:319-38.

Cite this article as: Mascardi V, Grecchi B, Barlascini C, Banfi P, Nicolini A. Effectiveness of temporary positive expiratory pressure (T-PEP) at home and at hospital in patients with severe chronic obstructive pulmonary disease. J Thorac Dis 2016;8(10):2895-2902. doi: 10.21037/jtd.2016.10.69
18. ATS Committee on Proficiency Standards for Clinical Pulmonary Function Laboratories. ATS statement: guidelines for the six-minute walk test. Am J Respir Crit Care Med 2002;166:111-7.

19. Lawati NA, FitzGerald JM. Acute exacerbation of chronic obstructive pulmonary disease. BCMJ 2008;50:138-42.

20. Osadnik CR, McDonald CF, Miller BR, et al. The effect of positive expiratory pressure (PEP) therapy on symptoms, quality of life and incidence of re-exacerbation in patients with acute exacerbations of chronic obstructive pulmonary disease: a multicentre, randomised controlled trial. Thorax 2014;69:137-43.

21. Kodric M, Garuti G, Colomban M, et al. The effectiveness of a bronchial drainage technique (ELTGOL) in COPD exacerbations. Respirology 2009;14:424-8.

22. Bellone A, Spagnolatti L, Massobrio M, et al. Short-term effects of expiration under positive pressure in patients with acute exacerbation of chronic obstructive pulmonary disease and mild acidosis requiring non-invasive positive pressure ventilation. Intensive Care Med 2002;28:581-5.

23. van der Schans CP, Postma DS, Koëter GH, et al. Physiotherapy and bronchial mucus transport. Eur Respir J 1999;13:1477-86. 